LA-UR- $96-1682 \quad$ CONF- $960690--7$

Author(s):

Submitted to:

\title{
Title: HIGH ENERGY NEUTRON RADIOGRAPHY
}

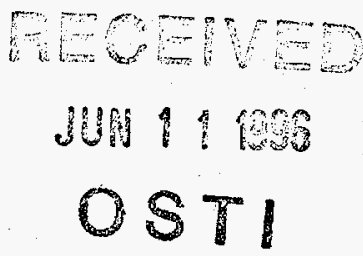

A. Gavron, K. B. Morley, C. Morris, S. J. Seestrom, J. L. Ulimann, G. J. Yates, and J. D. Zumbro

5th International Conference on Applications of Nuclear Techniques

Heraklion, Crete, GREECE

June 9-15, 1996

(FULL PAPER)

\section{Los Alamos}

NATIONAL LABORATORY

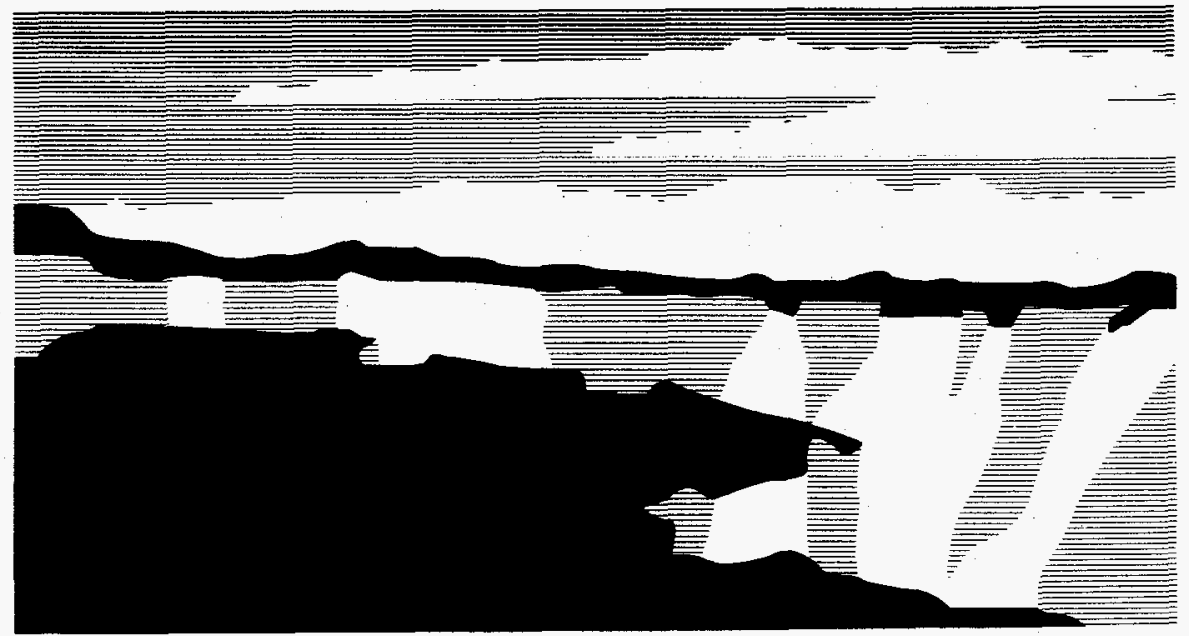

Los Alamos National Laboratory, an affirmative action/equal opportunity employer, is operated by the University of California for the U.S. Department of Energy under contract W-7405-ENG-36. By acceptance of this article, the publisher recognizes that the U.S. Government retains a nonexclusive, royalty-free license to publish or reproduce the published form of this contribution, or to allow others to do so, for U.S. Government purposes. The Los Alamos National Laboratory requests that the publisher identify this article as work performed under the auspices of the U.S. Department of Energy. 


\section{DISCLAMMER}

Portions of this document may be illegible in electronic image products. Images are produced from the best available original document. 


\title{
High Energy Neutron Radiography
}

\author{
A. Gavron, K. Morley, C. Morris, S. Seestrom, J. Ullmann, G. Yates and J. Zumbro \\ Los Alamos National Laboratory, Los Alamos, NM 87545
}

\begin{abstract}
High-energy spallation neutron sources are now being considered in the US and elsewhere as a replacement for neutron beams produced by reactors. High-energy and high intensity neutron beams, produced by unmoderated spallation sources, open potential new vistas of neutron radiography. We discuss the basic advantages and disadvantages of high-energy neutron radiography, and consider some experimental results obtained at the Weapons Neutron Research (WNR) facility at Los Alamos.
\end{abstract}

\section{INTRODUCTION}

Imaging with thermal neutrons is now a mature field, involving numerous applications $^{1}$, and several versatile detector systems ${ }^{2}$. The advent of high-energy spallation sources has opened new opportunities for imaging with neutrons at higher energies. These neutron energies range anywhere from epithermal, on up to hundreds of $\mathrm{MeV}$. High-energy neutron radiography differs significantly from thermal neutron radiography, in that the absorption and scattering cross-sections exhibit a more regular dependence on the nuclear mass $\mathrm{A}$. At energies above a few $\mathrm{MeV}$, the dominant factor in the neutron cross-sections is the area of the nucleus, which is proportional to $\mathrm{A}^{2 / 3}$. This Adependence is much slower that the equivalent dependence for photon absorption. As a result, one can obtain a much better contrast between light and heavy materials using neutrons, compared to photon radiography. As an example, we consider the contrast between ${ }^{238} \mathrm{U}$ and ${ }^{6} \mathrm{Li}$. The ratio of total neutron cross-sections is approximately 10 , whereas the comparable ratio of photon cross-sections is 150 . Lower energy neutrons provide even more contrast, but higher energy neutrons have the advantage of being much more penetrating. This implies that we should, in principle, search for the optimal neutron energy that provides the best compromise between the transmission rate and the signal-to-noise ratio. In practice, spallation sources that provide high-energy neutrons are usually span a large energy range. We can then consider what optimum energy range should be used for neutron radiography, and design the detector to optimize that energy range. We describe a simple model to do this in the next section. In subsequent sections, we present results of simulation calculations, detector designs, and some experimental results.

\section{DETERMINING THE OPTIMUM NEUTRON ENERGY}


The Weapons Neutron Research facility (WNR) at the Los Alamos Neutron Science Center (LANSCE) utilizes an $800 \mathrm{MeV}$ proton beam, impinging on a tungsten cylinder, approximately $7.5 \mathrm{~cm}$ long, and $1.5 \mathrm{~cm}$ radius. Typically, pulses of $2 \cdot 10^{8}$ protons arrive

every $1.8 \mu \mathrm{sec}$, bunched in macropulses approximately $800 \mu \mathrm{sec}$ long. 100 such macropulses are delivered every second. The neutron spectrum generated is presented in Figure 1. We note that the spectrum extends up to higher energies at the more forward angles.

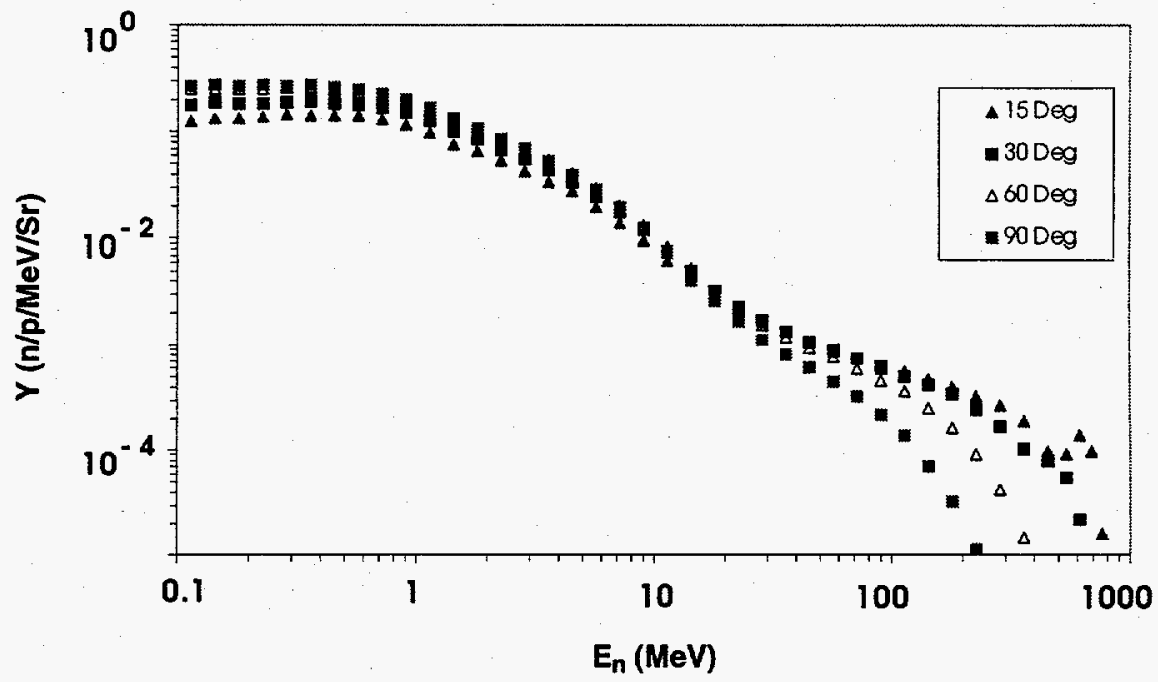

Figure 1. Spectrum of neutrons at WNR, Y, as a function of energy, for different angles relative to the proton beam.

At energies below $50 \mathrm{MeV}$ there is very little difference between the spectra at different angles. The neutron total crosssection decreases significantly over the same energy range. Consequently, the decrease in the number of neutrons at higher energies, is somewhat offset by the increase in the penetrability of these neutrons. To illustrate this, we consider the problem of imaging a $\mathrm{LiH}$ plate, covered by a uranium plate. A schematic of the setup is presented in figure 2. We assume that the uranium is $10 \mathrm{~cm}$ thick, and the $\mathrm{LiH}$ is 2.5 $\mathrm{cm}$ thick. A small cone ("defect"), $2 \mathrm{~mm}$ in base radius and $2 \mathrm{~mm}$ high is evident. This defect is the signal we wish to see. The relative magnitude of the signal generated by the defect is presented in Figure 3 as a function of the neutron energy (it

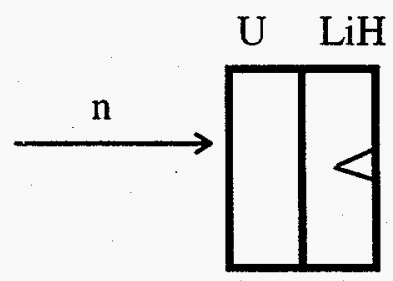

Figure 2: Schematic setup

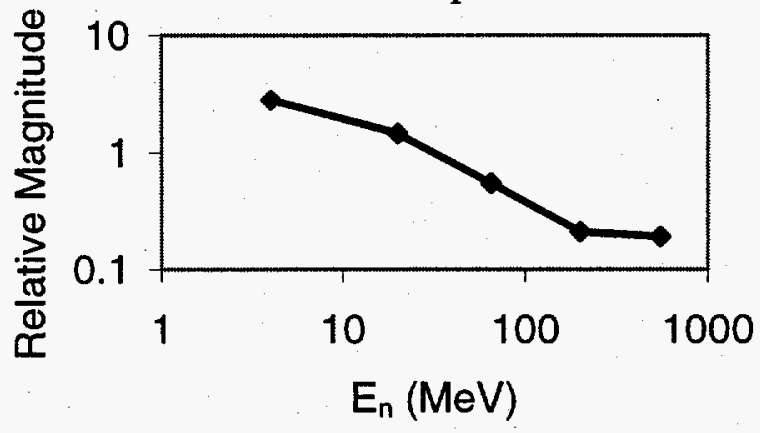

Figure 3: Relative magnitude of effect vs. energy 
is proportional to $\left.\sigma_{\mathrm{Li}} / \sigma_{\mathrm{U}}\right)$. We determine the time it takes the signal (i.e. the enhancement in the number of transmitted neutrons due to the defect) to reach the level of five standard deviations of the number of transmitted neutrons. The calculation is purely geometric, using an attenuation of $\mathrm{e}^{-\Sigma \mathrm{X}}$, where $\Sigma$ is the macroscopic total neutron cross-section, and $\mathrm{X}$ is the total thickness. The calcu-

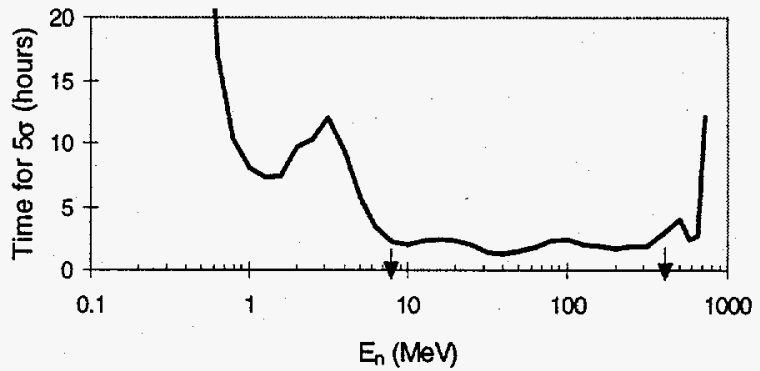

Figure 4. Time till signal rises above $5 \sigma$ of background lation does not consider the energy dependence of the neutron detector, nor does it consider the background due to scattered neutrons. The time is presented in figure 4 for a specific configuration at WNR, as a function of the neutron energy. We get the lowest time (i.e., best "Figure of Merit") between 8 and $400 \mathrm{MeV}$. This is a result of a tradeoff between the decreasing contrast (i.e. $\sigma_{\mathrm{Li}} / \sigma_{\mathrm{U}}$ ) and the increasing transmission as the neutron energy increases, weighted by the WNR flux. This result implies that if we were able to design a neutron detector with a flat efficiency curve, the ideal energy range would be 8 to $400 \mathrm{MeV}$. In fact, neutron detector efficiencies typically decrease as the energy increases. We will return to this topic later.

\section{OPTIMIZING THE CONFIGURATION}

There are several factors that need to be considered in a more detailed optimization on the radiography configuration. The first of these is the effect of scattering. MottSchwinger scattering together with small-angle elastic scattering contribute to blurring the image, since they cause a deviation in the straight-ray assumption we use. Although Mott-Schwinger scattering has an asymptocically infinite cross-section at small angles, we have calculated that $98.6 \%$ of the neutrons going through typical samples are scattered by an angle less than $10^{-3}$ degrees. Small angle elastic scattering likewise does not cause a significant blurring. Inelastic scattering is a different issue - at higher energies, a single incoming neutron can give rise (via the spallation and subsequent fission processes) to several lower energy secondary neutrons. These neutrons are hardly correlated with the direction of the incoming neutron and they produce a smooth ("gray") background.

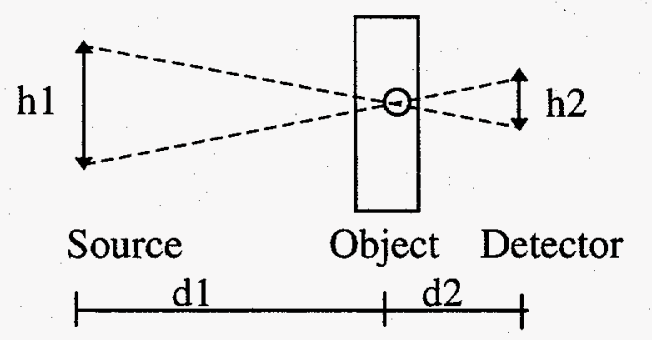

Figure 5: Image of source on detector This background increases the time necessary to obtain a statistically meaningful signal. The second effect is that of the sourceobject-detector geometry - if we are imaging a point defect in an object at a distance d1 from the neutron source, onto a detector at a distance of $\mathrm{d} 2$ behind the object, we produce an image of the source size $h 2=$ 
(d2/d1) h $\mathrm{h}$, where $\mathrm{h} 1$ is the source size (see figure 5). Thus, this consideration drives us to moving the detector as close as possible to the object. The problem is that the background due to secondary spallation and fission neutrons varies approximately as $\mathrm{d} 2^{-2}$ (the object serves as the "source" for these neutrons) whereas the signal due to the directly transmitted neutrons varies as $(\mathrm{d} 1+\mathrm{d} 2)^{-2}$. Therefore a better signal-to-noise ratio is obtained by increasing $\mathrm{d} 2$. Optimization of this process depends on various factors: The size of the object (it determines the spallation induced background), the size of the source (h1), the potential size of the defect to be imaged, and the resolution of the detector.

\section{PROOF OF PRINCIPLE EXPERIMENT}

On the WNR 15 degree flight path, the effective source size as seen from the detector is approximately $3 \mathrm{~cm}$ high by $5 \mathrm{~cm}$ wide. The flight path length to the detector station is approximately 80 meters. We performed a first "proof of principle" experiment in the summer of 1994. The object we imaged was comprised of $10 \mathrm{~cm}$ thick uranium, and a $\mathrm{LiH}$ disk that was $2.54 \mathrm{~cm}$ thick. We drilled several different size holes in the $\mathrm{LiH}$ - three holes through the $\mathrm{LiH}$ with diameters of 12,8 and $4 \mathrm{~mm}$ (top row in figure 6), and two holes half-way through $(1.27 \mathrm{~cm}$ deep) the $\mathrm{LiH}$ with diameters of 12 and $8 \mathrm{~cm}$ (bottom row in figure 6). In addition, a small hole (diameter of $1.75 \mathrm{~mm}$ ) was drilled half way

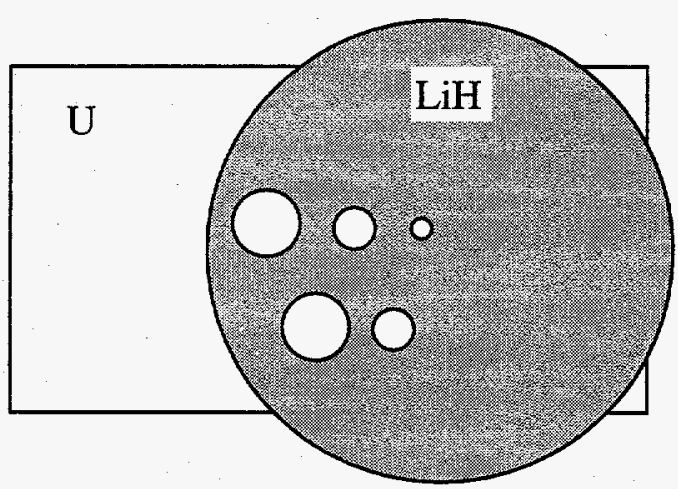

Figure 6: $\mathrm{LiH}$ disk and uranium configuration, showing the layout of the holes

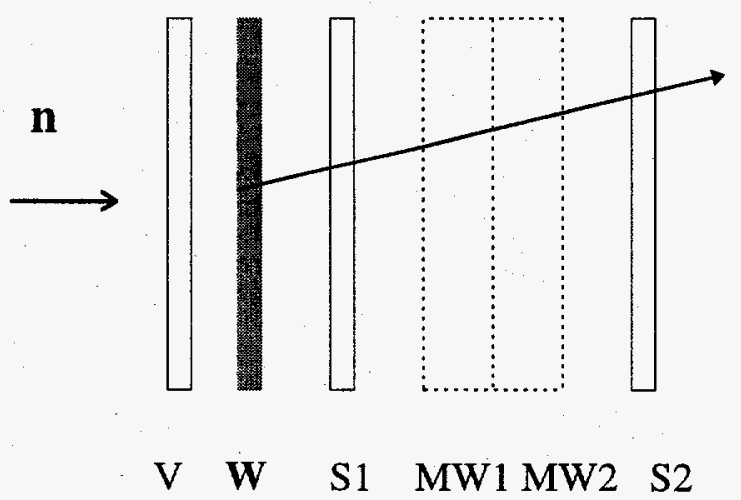

Figure 7: Schematic layout of detector system through $(5 \mathrm{~cm}$ depth) uranium brick. This was done to examine the effect of small-angle ("Mott-Schwinger") scattering on the produced image. The detector system we used is depicted in figure 7. The detector was positioned approximately three meters behind the $\mathrm{LiH} / \mathrm{U}$ object.

The principle of its operation is as follows (See fig. 7): A neutron interacts with the tungsten radiator $\mathrm{W}$, and a proton is ejected. A trigger is formed if the proton passes through the two scintillators $\mathrm{S} 1$ and $\mathrm{S} 2 . \mathrm{V}$ is an additional scintillator that vetoes charged particles produced before the $\mathrm{W}$ radiator. The $(\mathrm{x}, \mathrm{y})$ positions of the proton are determined in two adjacent multiwire detectors MW1 and MW2. (Circles on the proton track, in the drawing). These coordinates then determine the point of origin of the proton on the tungsten radiator (which is also the neutron posiion). The multiwire detectors have a 


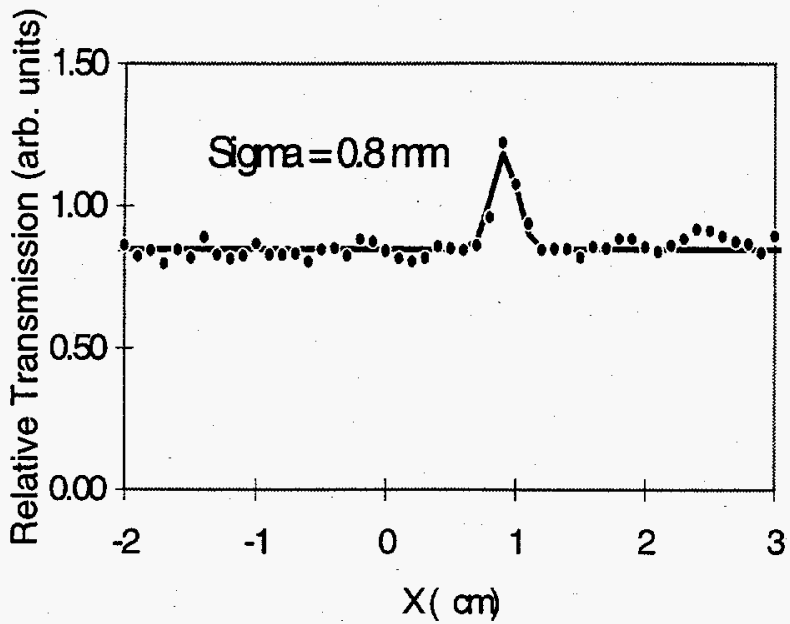

Figure 8: Image of transmission through pinhole.

\section{Experimental (WNR)}

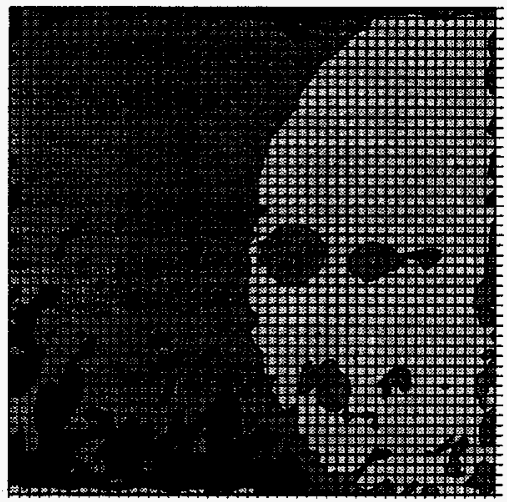

characteristic resolution of approximately $1 \mathrm{~mm}$ FWHM. We estimate the effective energy range of detected neutrons to be between 40 and 600 $\mathrm{MeV}$, due to the threshold of protons having sufficient energy to trigger S2. The efficiency of this detector was approximately $0.6 \%$.

The image of the pinhole is presented in figure 8 . The position resolution of the detector as determined by the width of the peak is 0.8 $\mathrm{mm}$. This confirms our conclusion that Mott-Schwinger scattering is not a significant issue.

\section{Simulated}

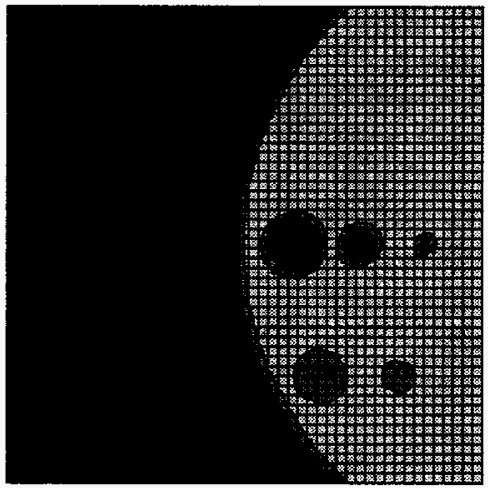

Figure 9: Image of the $\mathrm{LiH}$ disk shielded by the $10 \mathrm{~cm}$ thick uranium (compare to figure 6). The edge of the disk, as well as the existence of all 5 holes are clearly evident.

Figure 9 shows the integrated image obtained after 50 hours of data collection with the $\mathrm{LiH}$ in place. The image we present is obtained by dividing the number of counts in each pixel ( $1 \mathrm{~mm}$ in size) with the number of counts obtained with the $\mathrm{LiH}$ omitted. This process gets rid of systematic detection efficiency and beam intensity variations at different locations on the detector surface. For clarity, the simulated image does not include effects of statistics or background. The absence of the $\mathrm{LiH}$ is calculated to enhance the transmission by $4 \%$ - this is the contrast we observe for the fully-drilled holes in figure 9 .

\section{NEW DETECTOR DESIGN}

This experiment provided the information we needed to design a more efficient detection system. One of the major problems we encountered was the high dead time in our 
data acquisition system - of the order of $70 \%$. Since we need to move the entire experimental setup closer to the WNR source in order to obtain a higher counting rate, this was a major obstacle. It became obvious that we need an image-integrating detector, rather than a detector that processes individual neutron interactions. For the 1995 WNR experimental cycle, two detectors were developed. They will be described in detail elsewhere in this conference, but for completeness we provide a short review.

The first detector type is an extension of the existing MWPC detectors. The anode was a $2 \mathrm{~mm}$ thick copper plate that doubled as a radiator (converting neutrons to protons). It was divided into pads sized $0.25 \mathrm{~cm} \times 0.25 \mathrm{~cm}$ each. The protons, produced by neutrons, cause avalanches that are localized to adjacent pads. Charge on the pads is integrated over several seconds before reading it out through a gated FET to an integrating ADC. The pads are gated and read sequentially by the data acquisition system. The efficiency of this system is comparable in magnitude to that of the original system $-0.6 \%$. It is sensitive to neutrons above $40 \mathrm{MeV}$.

The second detector is built from scintillating optical fibers. The fibers are $5 \mathrm{~cm}$ long, and $0.25 \mathrm{~cm}$ in diameter, bundled into a cylinder with a diameter of $9 \mathrm{~cm}$. The fibers are imaged at the edge of the bundle using a lens, image intensifier, and a CCD to record the image. This detector produces a clear image as long as the recoiling protons do not traverse more than 1-2 fibers. This limits the detector to incoming neutron energies below 30 $\mathrm{MeV}$, which can be achieved by gating the image intensifier to a specific time window relative to the start time of the neutron pulse from the WNR target. The efficiency of this detector is expected to be of the order of $10 \%$.

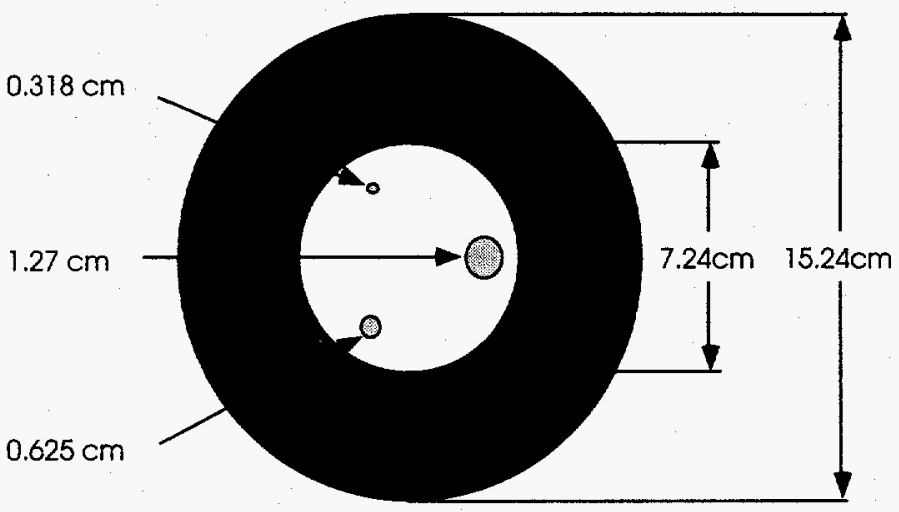

Figure 10: Uranium cylinder, polyethylene filling, and drilled holes through the polyethylene

\section{THE TOMOGRAPHY EXPERIMENT}

Tomographic imaging has been well established with electromagnetic radiation and with thermal neutrons. We designed an experiment to investigate its feasibility with high-energy neutrons. A uranium cylinder, 15.24 $\mathrm{cm}$ outer diameter, $7.24 \mathrm{~cm}$ inner diameter, $20.3 \mathrm{~cm}$ length, was filled with a polyethylene cylinder. Three off-center holes were drilled through the polyethylene, parallel to its axis (see figure 10). The diameters were $0.32,0.63$, and $1.27 \mathrm{~cm}$ Radiographs of the object were taken by a prototype integrating system, with 48 -by-48 pixel pads, covering 12.2 -by- $12.2 \mathrm{~cm}^{2}$. The cylinder was placed wire-chamber detector 20 meters from the WNR source, on the 30 degree (right) beam 

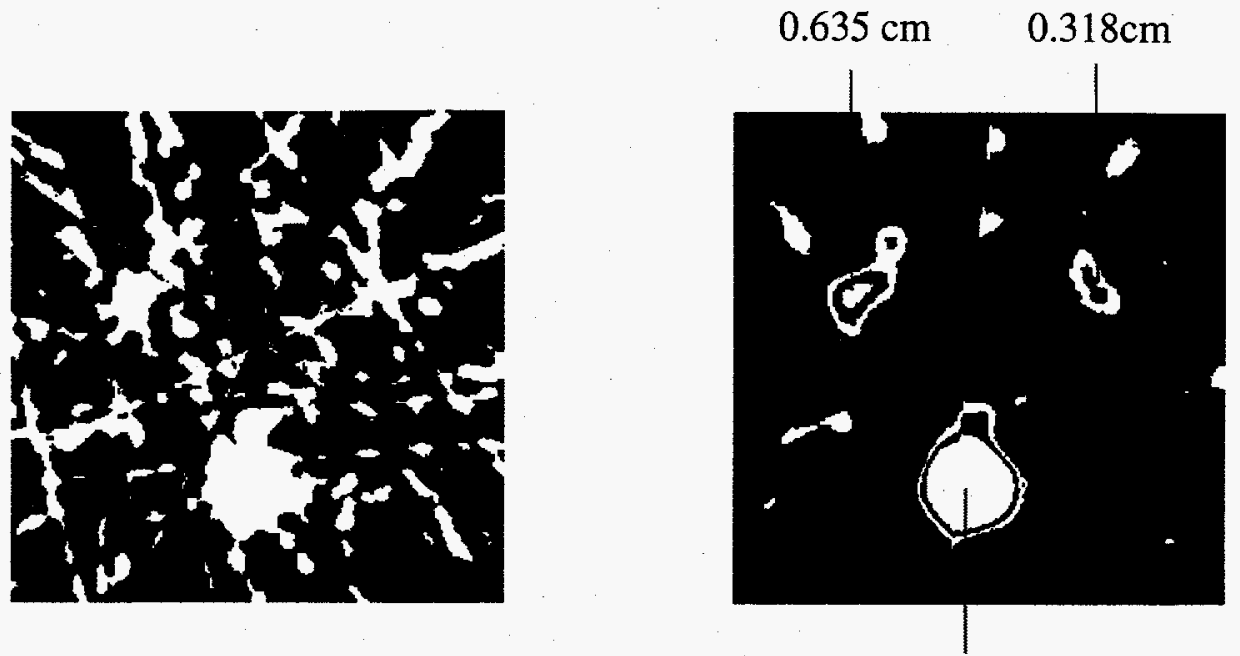

$1.27 \mathrm{~cm}$

Figure 11: Back-projection reconstruction of the defects in the cylinder. a) denotes the uncorrected reconstruction, b) depicts the contrast after introducing a threshold in the plot.

line at WNR. The detector was placed 2 meters downstream from the cylinder. One radiograph was taken with a polyethylene cylinder without holes, and nine radiographs were taken with the drilled plastic cylinder, by rotating the cylinder by 20 degrees between each exposure. Each angle took approximately 8 hours. The radiographs were formed by taking the ratio of images between the drilled cylinders and the blank cylinders. This was necessary to deal with systematic effects in the detector and the beam. Unfortunately, due to instabilities in the detector; the radiographs deteriorated over the course of successive angle measurements. However, after correction for these instabilities during the replay of the data, it was still possible to use tomographic reconstruction techniques to locate defects within the cylinder. The effects of all three voids are visible on this radiograph. The two larger holes are evident on the original (Figure 11, a) radiograph, and the third hole becomes evident as the threshold is increased in the plot (Figure $11, \mathrm{~b}$ ). We conclude that holes with a $3 \mathrm{~mm}$ diameter in a light material (polyethylene) are visible even when shielded by a total of $8 \mathrm{~cm}$ thick uranium. Obviously, more work needs to be done to obtain a stable beam environment and detector system.

\section{PRACTICAL APPLICATIONS}

Since high-energy neutron sources are not widely available, the question arises as to whether there is any practical alternative that is also reasonably cost-effective. For certain systems, with thicknesses up to approximately $200 \mathrm{~g} / \mathrm{cm}^{2}, \mathrm{a}^{252} \mathrm{Cf}$ source could become a viable alternative. Although a fission neutron is less penetrating than the WNR highenergy neutron spectrum, the contrast produced by the lower energy neutrons is much larger. In addition, fission neutrons can be detected at significantly higher efficiencies, of the order of $10 \%$. ${ }^{252} \mathrm{Cf}$ sources with small sizes are available from ORNL - a $10^{10}$.neu- 
trons/sec source can be contained in a steel capsule, $0.071 "(1.8 \mathrm{~mm})$ inner radius and 0.075 " (19 mm) long. A radiography setup for the initial WNR sample is presented in figure 12. The contrast between holes in the $\mathrm{LiH}$ and the surrounding material in the WNR experiment was $4 \%$. By comparison, the calculated contrast (using the MCNP4

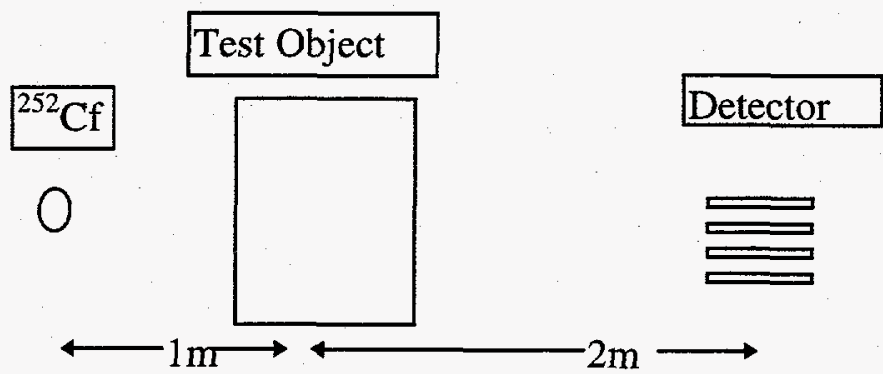

Figure 12: Setup of $\mathrm{LiH}$ test object with ${ }^{252} \mathrm{Cf}$ source neutron transport simulation $\operatorname{code}^{3}$ ) with a ${ }^{252} \mathrm{Cf}$ source is $33 \%$. Rate calculations assuming WNR neutron fluences at an 18 meters flight path and a detector with $0.6 \%$ efficiency (typical for a single plane conversion type high-energy neutron detector), and a $10^{10}$ neutrons/sec ${ }^{252} \mathrm{Cf}$ source in the configuration above, using a detector with $10 \%$

efficiency (typical for fission neutrons), indicate that with comparable exposure times one obtains statistically comparable images.

\section{REFERENCES}

1. G. W. Fraser, "Thermal neutron imaging", Proc International Conference on Neutrons and Their Applications, George Vourvopoulos and Themis Paradellis editors, Crete, Greece, June 12-18, 1994.

2. A. Breskin, R. Chechick, A. Gibrekhterman, V. Dangendorf and A. Demian, "Large-area high-resolutionthermal neutron imaging detectors", Proc International Conference on Neutrons and Their Applications, George Vourvopoulos and Themis Paradellis editors, Crete, Greece, June 12-18, 1994.

3. Judith F. Briesmeister, Editor, "MCNP - A general monte-carlo n-particle transport code", Los Alamos report LA-12625-M, 1993, version 4A.

\section{DISCLAIMER}

This report was prepared as an account of work sponsored by an agency of the United States Government. Neither the United States Government nor any agency thereof, nor any of their employees, makes any warranty, express or implied, or assumes any legal liability or responsibility for the accuracy, completeness, or usefulness of any information, apparatus, product, or process disclosed, or represents that its use would not infringe privately owned rights. Reference herein to any specific commercial product, process, or service by trade name, trademark, manufacturer, or otherwise does not necessarily constitute or imply its endorsement, recommendation, or favoring by the United States Government or any agency thereof. The views and opinions of authors expressed herein do not necessarily state or reflect those of the United States Government or any agency thereof. 